\title{
Racismo epistémico en el "sertão" del nordeste brasileño: un abordaje genealógico de la salud mental
} Epistemic racism in the brazilian northeastern "hinterland": a genealogical approach to mental health

\author{
Emilene Andrada Donato \\ Universitat Autònoma de Barcelona \\ Universidade Estadual de Ciências da Saúde de Alagoas
}

\begin{abstract}
Resumen
Este articulo trata del racismo epistémico en el sertão del nordeste de Brasil, entre finales del siglo XIX y principios del siglo XX. Responde a dos objetivos: 1) evidenciar ese fenómeno aplicado al territorio; 2 ) visibilizar personajes e influencias epistemológicas en el campo de la salud mental, estimulando más estudios. Dentro del abordaje genealógico se realizó una búsqueda bibliográfica y documental interdisciplinaria, interlocuciones con actores, análisis interseccional y poscolonial bajo la interface entre dos paradigmas del periodo: racial/mestizaje y territorio/sertão. Concluye que la racialización y subalternización encontradas se asocian al momento político de construcción de la identidad nacional, siendo relevantes las teorías psiquiátricas, como la degeneración y la eugenesia, que también influyeron en la psicología. Están demostradas colonialidades inherentes al mesianismo y al bandolerismo nómada. Se sugiere ampliar el término de científico a epistémico, revisiones de disciplinas y prácticas globales, desde el punto de vista de la descolonización epistémica y de la Reforma Psiquiátrica.
\end{abstract}

Palabras clave: Racialismo; Prejuicio Geográfico; Colonialismo; Salud mental

\begin{abstract}
This article presents epistemic racism in the "hinterland" of north-eastern Brazil in the late nineteenth and early twentieth centuries. It responds to two objectives: 1) to evidence this phenomenon applied to the territory; 2) to make visible epistemological personages and influences in the area of mental health, stimulating more studies. Using the genealogical approach, interdisciplinary bibliographic and documentary analysis, interlocutions with actors, intersectional and postcolonial analysis were conducted according to the interface between two paradigms of the period: racial/mestizaje and territory/ hinterland. It concludes that the racialization and subalternization found are associated to the political moment of national identity construction, being relevant the psychiatric theories, such as degeneration and eugenics, which also influenced psychology. Colonialities on messianism and nomadic banditry are demonstrated. It is suggested to amplify the term from scientific to epistemic, reviews of disciplines and global practices, in the direction of epistemic decolonization and Psychiatric Reform.
\end{abstract}

Keywords: Racial Prejudices; Geographic Discrimination; Colonialism; Mental health 


\section{INTRODUCCIÓN ${ }^{1}$}

El presente artículo es parte de una investigación con enfoque genealógico sobre la salud mental brasileña en la subregión sertão ${ }^{2}$ de la región nordeste del país. Evidencia el racismo epistémico como un fenómeno relevante durante finales del siglo XIX y mediados del siglo XX, debido a la temática racial en debate por diversos sectores de la sociedad. Tras la abolición de la esclavitud y la transición del régimen monárquico al republicano, los políticos e intelectuales perseguían una conformación de la identidad nacional influenciados por filosofías como el positivismo, el evolucionismo y el darwinismo, y las ciencias europeas. Puesto que los papeles de la medicina higienista y de la psiquiatría son relevantes, se concentra en este último, que influyó más directamente en la constitución de la psicología nacional brasileña. Así también, aparecen algunos personajes y sus discursos más directamente relacionados con el territorio.

En ese sentido, se acerca a las posibles relaciones entre dos grandes paradigmas de la época estudiados más ampliamente por las ciencias sociales mestizaje y sertão-, trayéndolos al campo de la salud mental con una mirada crítica interseccional y poscolonial. Tal y como fueron concebidos, eran impedimentos a la sociedad civilizada deseada por las élites, siendo la intervención racial una medida profiláctica. Considerando que aparte del debate sobre el problema de la estructuración y ocupación social de los grandes centros urbanos, y específicamente en relación con los negros ex esclavizados, el argumento central indica que esta región funcionó como suelo fecundo a las teorías racialistas atribuidas al mestizo brasileño, donde la psiquiatría aplicó teorías colonialistas de la degeneración y de la eugenesia.

Los siguientes elementos se incorporan en la discusión: como lugar diametralmente opuesto de los referentes iluministas costeros, el determinismo racial aplicado en el campo de la salud, el mesianismo y el bandolerismo nómada en eventos notables, razones de fijación en el imaginario social y cultural sobre el ambiente sertanejo. Con ello se pretende: 1) evidenciar el racismo epistémico sobre el sertão con un enfoque en el nordeste a partir de la psiquiatría brasileña en ese período; y 2) visibilizar actores centrales e influencias epistemológicas en la construcción de la psicología y de la salud mental en el país.

\footnotetext{
${ }^{1}$ Este trabajo ha sido realizado en el marco del programa de doctorado "Persona y Sociedad en el Mundo Contemporáneo", de la Universitat Autònoma de Barcelona (España) y como doctoranda visitante en el "Centro de Estudos Sociais" de la Universidade de Coimbra (Portugal).

2 Juan Manuel Fernández (2013, p. 24), apunta que el vocablo regional sertões no tiene equivalente en el idioma español. Se afirma aquí su singularidad, manteniéndolo así en portugués, incluso para su pueblo nombrado como sertanejo.
} 
Como el análisis social impulsa conocer a fondo nuestros contextos, urgen nuevos diálogos con la historia que estructura nuestras realidades. Reconstituir el camino de la colonización bajo las singularidades de los territorios nos ofrece una forma de encontrar los efectos perdurables del legado colonizador, o sea, las colonialidades en nuestras culturas, produciendo comprensión a partir de otros sentidos sobre problemas complejos y estructurales. Así, alrededor de la idea de raza, autores decoloniales señalan que se activan modos de organización de la vida en sociedad, la gestión y el control sobre los cuerpos, la construcción del conocimiento, el mundo del trabajo, relaciones sociales, entre otros aspectos, teniendo en cuenta "topologías" del poder, saber y ser, relacionadas con los espacios, lugares, dentro de una cadena interconectada de jerarquías globales. Es posible observar, por tanto, las dimensiones epistémica, lingüística, de género, sexualidad, espiritualidad, ambiente, subjetividades (Maldonado-Torres, 2009; Quijano, 2005; 2009).

Entre lo que está calificado como universal y lo que es singular, en cuanto al territorio en concreto, es cierto que sertão es ampliamente considerado en la literatura casi hasta el siglo XIX como referente a cualquier área del país dirigida hacia el "interior". Su etimología, con origen colonial, según Durval Muniz de Albuquerque Júnior (2019, p. 21), se remite a la "Crónica del descubrimiento y la conquista de Guinea" (1452-53): "tierras que se adentran en el interior del continente africano, inaccesibles para los navegantes portugueses". Del primer diccionario de la lengua portuguesa (1728): "interior, corazón de las tierras, opuesto al mar y a la costa”, descripción que subraya las paradojas geográficas y simbólicas. Sin embargo, en el siglo XX se ha convertido en una categoría "capturada gradualmente por el discurso regionalista del nordeste" a través de la literatura, la política, los medios de comunicación, la cultura y el arte, y, en 1969, oficializada por el Instituto Brasileño de Geografía y Estadística (IBGE).

Curiosamente, es desde los campos de la literatura y de la historia la elaboración de la llamada "psicología del universo del sertão", no observándose tal mención en el campo de la psicología. Se remiten a ésta las representaciones de figuras como "el jagunço, el cangacero y el beato", guiados por "polaridades extremas de la psique del sertanejo", con capacidades desde la "fe más irrestricta hasta la violencia más sangrienta”. Un sertão y un sertanejo históricamente ideados como "incapaces de racionalidad, llevados por las pasiones y la creencia más primitiva, carentes de educación y racionalidad" y "aún hoy, la gente del nordeste, especialmente los sertanejos, son vistos como poco racionales en sus decisiones (sobre todo políticas)", y aficionada al mesianismo y al populismo (Albuquerque Júnior, 2009, p. 30). 
Distinto a las aspiraciones modernas, la fuerza imagético-discursiva de esta zona fijada como "bárbara", "primitiva” y "subdesarrollada" atravesó el tiempo y fue generalizada para las demás subregiones con características diferentes del nordeste, presentadas en la figura 1. Las vulnerabilidades quedaron cristalizadas en el suelo semiárido, la sequía, la pobreza, la baja educación, la violencia, el éxodo rural y la migración de la población hacia el sureste durante el siglo XX, que produjeron la periferización de los sujetos y estereotipos de subalternidad. Estos componentes se han convertido en racismo manteniéndose hasta el presente, hecho analizado por Luciano Amaral Oliveira (2011), y en discursos que lo mantuvieron en una posición inferior a las regiones sur y sureste, fuertemente interpretada como una región subalterna relacionada precisamente con el hecho de ser un "pueblo mestizo" (Pinto, 2017).

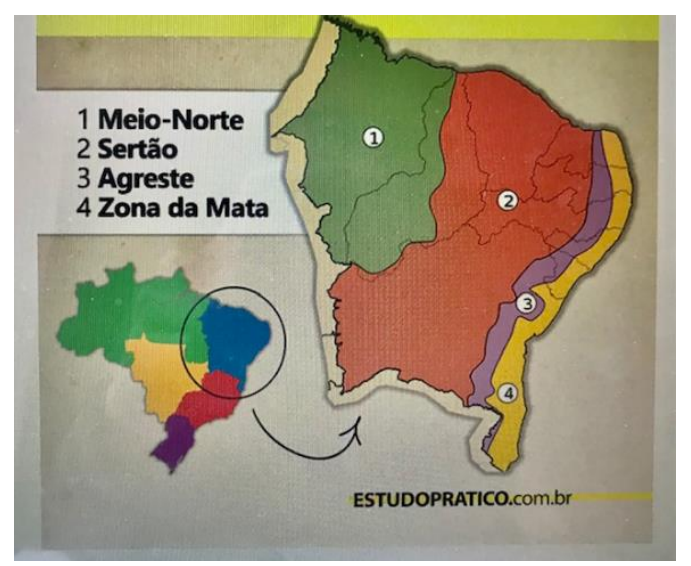

Figura 1. Sertão en rojo identificado con el número 2 Fuente: Polon (2019).

No obstante, se consideran tales razones mirando las huellas de los acontecimientos. De degenerados a enfermos, la concepción moralista sobre los mestizos tuvo cabida sobre la región y, como se verá, se consolidaron perjuicios duraderos. Los discursos de celebridades como Raimundo Nina Rodrigues (18621906), psiquiatra y antropólogo del nordeste, y Euclides da Cunha (1866-1909), ingeniero militar y periodista del sureste, responsable por la obra literariocientífica magistral sobre los "sertões" del país, además de los procedentes de la región, siguen esta línea, de modo que son perceptibles sus vestigios hasta la actualidad y repercusiones dentro del paradigma biomédico, psicopatologizante, excluyente y asilante. 


\section{CONSTRUCCIÓN TEÓRICA Y METODOLÓGICA}

La genealogía foucaultiana como un abordaje de análisis histórico articula los objetos en el ámbito de los discursos, relaciones de saber y poder, y subjetividad. Se aleja de la noción de "origen" (comienzo) y considera las nociones de "proveniencia" (proliferación de los acontecimientos) y "emergencia" (flujo de fuerzas en juego), sin pretender reconstituir una mirada lineal, sino mostrar sus discontinuidades y singularidades en relación con los hechos del presente. Proporciona, por tanto, una sensibilidad estratégica sobre el tejido social para enfrentar y desmontar dispositivos de normalización, objetivación y subjetivación constituidos históricamente, de manera documentada (Foucault, 2012; Prado Filho, 2017). Así, interesa volver al momento en que el sertão fue utilizado por la psiquiatría en la construcción del conocimiento bajo los códigos raciales y morales, con efectos en disciplinas como la psicología, pensando en contribuciones actuales al campo de la salud mental.

Se incorporó, además, la perspectiva de la "insterseccionalidad" yuxtaponiendo los marcadores sociales territorio y raza, particularmente para pensar nuestra realidad. Esto se debe a que el sistema de dominación de los territorios colonizados fue operado por la lógica racialista manifestada entre otros mecanismos por la inferiorización de actores locales y sus conocimientos, y articulado por epistemologías hegemónicas del norte global, con efectos sobre nuestro pensamiento aún vigente. Considerando que adicionalmente los marcadores sociales actúan conjuntamente, la experiencia del racismo es inseparable de otras estructuras como las de cisheteropatriarcado y capitalismo, como sostiene Carla Akotirene (2019). A su vez, cabe destacar que son fruto de los colonialismos y, por tanto, se utiliza la mirada poscolonial.

Para ello se procedió con búsquedas bibliográficas en buscadores virtuales como Scopus, Psychiatry on line Brasil, BVS- PSI y Scielo, en tres etapas. En la primera, fueron relacionados los descriptores Sertão y psiquiatría, psicología, salud mental. Luego, la obra "Os Sertões", y salud mental, psicología y psiquiatría; y, en la tercera, sertão, decolonialidad y poscolonialismo, para desarrollos en el sentido del debate paradigmático. De la búsqueda, se destacan algunos puntos. El primero se refiere a la invisibilidad científica del sertão dentro de la psicología y la salud mental, contrariamente a lo que sucede en otras áreas del conocimiento. Las producciones se ubican, en general, en el campo de la historia, la sociología y la antropología, especialmente relacionadas con este libro "Os Sertões", que resulta relevante, pues ocupa el estatus de "Biblia de la nacionalidad" brasileña (Cunha, 2003, p.16).

Enlazándolo con psiquiatría/salud mental, se observó el segundo punto: el fenómeno del "mesianismo", reportado en la narrativa, es señalado con un nú- 
cleo esencialmente psicopatológico. El tercer punto se refiere a los trabajos en la psiquiatría/historia de la ciencia de la salud producidos de forma destacada por otras regiones de Brasil y no por la nordeste. Se observaron temas que tratan de la historia involucrada con las áreas "psi" (psiquiatría, psicología, psicoanálisis), y Andrea de Alvarenga Lima y Adriano Furtado Holanda (2010) ya indicaban más producciones por otras regiones y el patrón aún se mantiene, a pesar del impacto de las políticas de interiorización de universidades y centros de formación en la década pasada. Este estudio historiográfico nos lleva a los períodos de construcción de los modelos de atención en salud mental en Brasil, indicando entre 1890 y 1966 cuando se dio la constitución y consolidación de la psiquiatría.

Así también, en Alessandro de Oliveira dos Santos et al. (2012), es posible notar la importancia de la psiquiatría y el papel relevante de Nina Rodrigues para la constitución de la psicología. Al dividir en tres fases la construcción del pensamiento psicológico brasileño, en lo que respecta a las relaciones étnico-raciales entre finales del siglo XIX y principios del siglo XX, la Escuela Nina Rodrigues es señalada como consolidadora de las investigaciones de las características psicológicas de los esclavizados y ex esclavizados, ofreciendo elementos para la configuración del negro como sujeto psicológico, configurándose dentro de un periodo "biológico-causal". En esta fase se detuvo el presente trabajo para mirar el caso de los sertões allí. Las otras dos fases se dividen, por un lado, entre 1930 y 1950, cuando tuvo lugar el debate sobre la construcción sociocultural de las diferencias y la deconstrucción del determinismo biológico de las razas, un momento "culturalista" en la constitución del campo de la psicología y la psicología social en Brasil; y, por otro lado, a partir de 1990, con los estudios sobre el blanqueamiento y la blanquitud, un periodo denominado "relacional". Se entiende que estos discursos y prácticas de la psicología a lo largo de su historia deben ser observados hacia el paradigma biopsicosocial, que rompe con las formas biologicistas, tecnicistas y ahistóricas para producir realmente salud.

El cuarto punto, la producción científica en el campo de la salud mental sobre el sertão, además de reducida, está más enfocada en evaluaciones de dispositivos gubernamentales, como acciones, servicios y políticas públicas. Se recomienda la inclusión en los análisis del campo la intersección entre los marcadores sociales, puesto que los datos socioeconómicos relativos a nuestras vulnerabilidades en relación con otras regiones siguen siendo substancialmente discrepantes y revelan alertas históricas sobre estos aspectos, tal y como se recoge en el informe del Instituto Brasileiro de Geografia e Estatística - IBGE (2019). Dada la complejidad del objeto y la escasez de trabajos constatada en 
el campo de la salud mental, se trabajó con una sumatoria bibliográfica de la literatura, historia, geografía, educación, sociología, antropología, medicina y psicología, además del periodismo virtual, y se establecieron interlocuciones con actores del mundo académico.

\section{Algunas conformaciones conceptuales y epistemológicas}

Se ha observado la importancia de las epistemologías críticas para comprender las colonialidades en estos puntos. En la fila de discusión, algunos conceptos asumen aquí centralidad como es el caso de raza, colonialidad interna y racismo epistémico, este último presentado en la próxima sección. La raza es considerada como una construcción política y social que organiza, como categoría discursiva, un sistema de poder socioeconómico, explotación y exclusión que es el racismo (Hall, 2003). Además, se subraya el vaciamiento del carácter problemático de la discusión racial relacionada con la diferencia cultural como herencia de la idea de ideología nacional en América Latina, acercada poco al campo poscolonial, como ha sido tratado por Leticia Cesarino (2017). Es decir, se configura como un mecanismo de invención colonialista sobre regiones subalternizadas, que conformó procesos similares en varios países, como también es el caso de Colombia y Argentina (Bolívar, n.d.; Fernandéz, 2013), y generó implicaciones para las poblaciones mestizas.

Así, la raza lleva ambigüedades e insuficiencias en la lista de pautas actuales de identidad, y por lo tanto lo utilizo aquí porque marca el periodo analizado. Sin embargo, por alusión a un "Otro", diferente en la alteridad, hasta cierto punto se acerca a lo que el militante político y psiquiatra Frantz Fanon (2008) denominó la "zona del no ser" cuando se refirió a los negros. Tal racialización sobre el sertão y el sertanejo produjo representaciones en el imaginario social, especialmente por la fuerza del pensamiento médico-higienista a partir de los informes científicos de viajes para conocer el interior del país. Julio Paternostro, en 1945, por ejemplo, consideró el sertanejo como "paria de la civilización", conformado por la "tradición" a un nivel de vida entre los siglos XVIII y XIX y un "etnocentrismo innegable" (Lima, 2009). Con ello se demuestra la importancia del concepto de "colonialidad interna" que traduce esa fuerza porque:

Tiende a seguir líneas geográficas: divisores como la costa/interior y lo rural/urbano han sido históricamente centrales tanto para las diversas "interpretaciones de Brasil" como para las políticas concretas propuestas por el estado. Vale la pena señalar cómo estos divisores producen internamente efectos de colonialidad similares (e interconectados con) aquellos observados externamente. (Cesarino, 2017, p. 87; énfasis propio) 
Se ve que para discutir los racismos importa movernos por escenarios y otras épocas de América, África, Asia, aparte de desplazamientos internos como sujetos políticos y afectivos, dada la confrontación con la construcción social de nuestra propia historicidad y noción de mundo y humanidad. De ese modo, bajo el entendimiento de los "poscolonialismos", en plural, porque se requieren análisis históricos situados, Portugal ha sido un "colonizador subalterno". Los grandes imperios de Europa occidental del siglo XVII ya se habían trasladado a Francia e Inglaterra y, entre otras cosas, nos proporcionó paradojas. De las violencias por genocidio y epistemicidio, la última es considerada más vasta, dada la pretensión deliberada de subalternizar y subordinar prácticas y grupos sociales que obstaculizaron el emprendimiento capitalista (Cesarino, 2017; Santos, B., 1995; 2003).

En torno a los proyectos antirracista, antipatriarcal y/o anticapitalista, y de una reciente ola de protestas en el mundo, se observó el reposicionamiento de sujetos y de una cierta "europeidad" crítica tradicionalmente acomodada en espacios de conocimiento y poder en nuestro campo. Además de los modos europeos de socialización, nos fue extendida la formación "absolutamente ortodoxa, convencional, experimental, positivista", sobre la cual el pensamiento poscolonial viene siendo una alternativa teórico-metodológica y crítica no solo para revisión del conocimiento, sino también para la sociedad, porque ofrece formas de resistencia social cómo herramienta política, en concordancia con Lupicinio Iñiguez-Rueda (2010, p. 694). La Salud Mental Global como campo emergente también viene reuniendo a especialistas y actores sociales que intervienen directamente en la salud pública y determinan objetivos del gobierno, políticas de reforma institucional y medidas preventivas. A su vez, conforme a Tiago Pires Marques (2018), pretende entender las formas contemporáneas de sufrimiento en términos de procesos cada vez más globalizados y secularizados de las economías capitalistas.

En esta tónica, y con mucho retraso, el tema del colonialismo y sus efectos destructivos aparece también en una carta abierta firmada por más de 100 psiquiatras británicos. Se pide al Royal College of Psychiatrists (RCPsych) respuesta al que llamó "racismo sistémico" (que incluye el tipo epistémico) y la discriminación en todo el sector. Se condenó la historia de la profesión por ignorar no solo los daños y perjuicios a los pacientes, sino también por concebir culturas "psicológicamente primitivas". Lejanos de "supersticiones retrógradas", incluso apunta que concibió la creación de una comisión integrada por usuarios de servicios y especialistas en "racismo institucional y colonialismo" para revisión de todos los planes de estudio de formaciones y las pautas prácticas porque: 
La psiquiatría, la psicología y la psicoterapia están profundamente arraigadas en las raíces históricas a través de las cuales los sistemas y estructuras sociales creados por la colonización, la esclavitud y la explotación económica fueron institucionalizados e incorporados a nuestras formas de vida y a las percepciones de los demás. Históricamente, la psiquiatría se ha coludido con el asesinato en masa eugenésico en la Alemania nazi y ha calificado de psicóticos a los manifestantes por los derechos civiles y a los disidentes políticos. Hasta el día de hoy, continuamos encarcelando a personas negras de manera desproporcionada y obligándolas a recibir tratamiento. Además, si es negro, es más probable que muera bajo medidas restrictivas cuando recibe atención de salud mental que si es blanco. (“Carta-Aberta contra o Racismo...", 2020, énfasis propio)

También cabe mencionar que, observadas estas terminologías utilizadas, parece no haber consenso en torno al uso del término racismo científico o epistémico, siendo notorio entre los teóricos latinoamericanos de lengua española la predilección por “epistémico”, y “científico” por los brasileños. Aparentemente, los países vecinos mencionan más otros pueblos como los originarios, contribuyendo al significado más ampliado, mientras la racionalidad científica específicamente está asociada a más estudios de los negros en Brasil. Además, las teorías raciológicas procesadas internamente los tomaron como objeto privilegiado en vista del gran numero de esclavizados y del impacto urbano en ese periodo sobre todo de los africanos y sus descendientes las grandes urbes. A considerar el "mestizaje" entre tres razas y sus distintos saberes, en este trabajo se ha dado la preferencia a la adopción ampliada de "epistémico".

En función de lo expuesto, entonces: ¿cómo movilizar las prácticas discursivas en el borde del interés académico integrando, finalmente, los temas referentes a los colonialismos y sus colonialidades en el campo de la salud mental?; ¿qué efectos producen el silenciamiento sobre esas cuestiones? A juicio de la tesis de Albuquerque Júnior (2009) titulada “La invención del Nordeste y otras artes", y de la lógica de la colonialidad interna, presentada a continuación, la propia región necesitaría comprender cómo refuerza un cierto status quo subalternizado que intenta combatir moviéndose y produciendo otros sentidos.

\section{RACISMO EPISTÉMICO Y LA INFLUENCIA DE LA MEDICINA EN LA PSICOLOGÍA}

Como el campo que problematiza los colonialismos es emergente y contrahegemónico, este apartado pretende acercarse brevemente a la construcción del término para luego incorporarlo al campo psicológico. El tipo de racismo “epistémico", abordado por Ramón Grosfoguel (2016, p. 39), presta atención a la idea de "raza" sistematizada por la lógica del mundo moderno-colonial y las consecuencias para el pensamiento, importando las influencias de la religión 
cristiana, la filosofía cartesiana y la economía capitalista. Por lo tanto, cabe recordar que España, desde la universalización de la identidad de los pueblos originarios americanos como "indios", cuando Colón imaginó que había pisado las "Indias Occidentales" en 1492, a la cristianización y explotación de los cuerpos y fuerza de trabajo bajo la justificación de criaturas "bárbaras" y sin alma, fue observada de cerca por otras partes de Europa. Es un hito importante que gira en torno del tribunal de Valladolid (1552) que, restableciendo el alma a los diferentes, serían exentos de la esclavitud, pero en la coercitiva "encomienda". Así, los africanos fueron despojados de humanidad y obligados a trabajar por más de 300 años en la mayor "empresa capitalista" de la modernidad.

Con ello se concibió el "racismo institucional" como un "principio organizador de la división internacional del trabajo y de la acumulación capitalista a escala mundial", complementando el viejo "racismo religioso", sospechoso de la humanidad de otros pueblos no europeos. En América, el antiguo antisemitismo religioso se transformó en antisemitismo racial, continuando la aniquilación del conocimiento de los dominados, el "genocidio/epistemicidio". La racialización se produjo con la marcación de cuerpos por color de la piel, prácticas étnicas, lingüísticas, rasgos religiosos y culturales. También con quema de libros en la conquista de Al-Andalus en el siglo XIII contra judíos y musulmanes, y de cuerpos vivos de mujeres acusadas de brujería, pues justamente era por la tradición oral y generacional la transmisión del conocimiento indoeuropeo. La esclavización de los africanos prohibió sus cosmologías y la propia producción de conocimiento, basada en la "inferioridad social biológica" creada por los europeos. De la "falta de inteligencia" en el siglo XVI se avanzó a "los negros tienen el coeficiente más bajo de inteligencia", o sea, se operó una lógica de "inferioridad epistémica”, por lo que Grosfoguel (2011, 2016, p. 40) configura el racismo epistémico o epistemicidio.

Como reflejo de ello, la ciencia pasó a legitimar en los cuerpos africanos indistintamente la neurocirugía de lobotomía y los comparó con el europeo "leucotomizado", es decir, una actualización del "primitivo", incluso desde los exámenes psicológicos bajo parámetros basados en la cultura europea (Fanon, 2020 , p. 235). Como consecuencias de siglos de dominación, se nota nuestra actualidad aún racista, y por eso Silvio Almeida (2019), entre otros autores, entiende el racismo como estructural, no arcaico, pues estructura las relaciones operando los tipos individual e institucional en Brasil. Hace patente que "el horror de las consecuencias coloniales es siempre más concreto que el horror de sus causas", como lo hace notar Boaventura de Sousa Santos (2020, p. 169). Es posible observarlas relacionadas con la raza/color en la "periferización" de 
grupos sociales más allá de los centros urbanos y precarización de sus condiciones de vida, en consecuencia, en los datos de desigualdades también sanitarias, en la manutención de estructuras de confinamiento y no de rehabilitación como el asilo y en cuanto el prohibicionismo moral en materia de drogas, en la ultra medicalización de las emociones, en el acceso limitado a los espacios de conocimiento y poder, en las violencias publicadas en las noticias cotidianas.

De ese modo, las filosofías del siglo XIX amparadas en la ciencia jerarquizaron y clasificaron socialmente a naciones enteras como inferiores y sub o no humanas. En ese periodo, los médicos sanitarios y los psiquiatras fueron los primeros y principales interesados en la supuesta "pureza racial", por ello también se subraya la diferenciación entre razas humanas, la mejora y rescate de la población. En Brasil, el centro del poder político estaba ahora en el sureste, y se fundó la Sociedad de Eugenesia de São Paulo (1918), pionera en América Latina; la Liga Brasileña de Higiene Mental (1923), que con el objetivo de reformas en la asistencia psiquiátrica adoptó ideas alemanas, francesas y estadounidenses (dando oficialmente al racismo "estatuto científico", según Walter Melo, 2004, p. 188); el Congreso Brasileño de Eugenesia (1929); la Comisión Central Brasileña de Eugenesia, con un Boletín de Eugenesia. La "Liga PróSaneamiento" (1918), entrecruzó el sanitarismo y la eugenesia preocupándose, en general, por la "eugenesia preventiva" (control de los factores disgenésicos a través del saneamiento); la "eugenesia positiva" (educación y control de la procreación de los capaces); y la "eugenesia negativa" (evitación de la procreación de los inútiles), organizando reformas de las políticas de salud pública (Caponi, 2014; Santos, R., n. d.; Seixas et al., 2009; Wegner, 2017).

En el nordeste brasileño, de las Ligas de Higiene Mental en algunos estados se destacó la de Pernambuco (1933), liderada por Ulysses Pernambucano (18921943), con una posición contraria a la orientación nacional. A pesar de su formación kraepeliniana, por la influencia del sociologo Gilberto Freyre, adoptó las perspectivas sociológica y antropológica que contribuyeron, por ejemplo, para que el control de la policía sobre los cultos de "candomblé" (religión afrobrasileña) migrara al Servicio de Salud Mental, como un avance en medio de las teorías racialistas que circulaban. Además, posibilitó la implantación del primer polo de psiquiatría social en Brasil, y de los primeros servicios públicos clínico-psiquiátricos, una escuela para personas con discapacidad mental y también el Instituto de Psicología, donde se llevaron a cabo las primeras adaptaciones y estandarización de exámenes psicológicos extensivos para la realidad brasileña (Medeiros, 1999; Melo, 2004).

Como se observa, la psicología se desarrolla a partir de la psiquiatría de forma orgánica, atravesada por las relaciones étnico-raciales en la sociedad. A partir 
de los años 30 -coincidiendo con la fase culturalista del pensamiento social brasileño, muy impactada por Gilberto Freyre (1933/2006), mientras que los estudios mostrarán más diagnósticos de enfermedades mentales (esquizofrenia, psicosis maníaco-depresiva) y toxico-infecciones (sífilis y alcoholismo) en personas negras y mestizas. En la revisión de Alessandro de Oliveira dos Santos et al. (2012) - se intensificará el debate para deconstruir el determinismo biológico de las razas, figurando entre los principales estudiosos hasta 1950 nombres como: Raul Briquet, Arthur Ramos, Donald Pierson, Virginia Leone Bicudo, Aniela Ginsberg y Dante Moreira Leite, responsables de los primeros cursos académicos de psicología social y de la delimitación del campo de la Psicología en Brasil, que también merecen ser repasados dentro de una agenda relativa a los territorios en relación al periodo enfocado.

\section{RACISMO EPISTÉMICO EN EL SERTÃO DEL NORDESTE}

Con la pretensión de construir una nueva nación, dentro del ambiente político e intelectual, había por tanto muchos personajes interesados en asegurar la viabilidad de una civilización moderna, tratando de resolver el problema de las razas consideradas inferiores. En general, las controversias discursivas reflejaron la imposibilidad de reproducción estricta de las teorías importadas, lo que fue notable en el ámbito de la eugenesia, a partir de los años veinte. Pesaban contra Brasil estigmas de la degeneración progresiva, la hibridez moral que atestiguaba la fragilidad de carácter, también llamando la atención a Juan Manuel Fernández (2013), la falta de "solidaridad" mediante una "moral rudimentaria" de los costeros en relación con la posición antitética de la costa-sertão. Para Cunha (2003, p. 109), el sertanejo estaría vivo en un lugar donde las características topográficas con nombres indígenas serían "bárbaras y extrañas" $\mathrm{y}$, aunque en algún momento afirme que "el sertanejo es un retrógrado, no un degenerado" (p. 115), considera que su fuerza física es rescatable. Afirmó que

Su inestabilidad en cuanto complejo de factores múltiples y diversamente combinados, sumado a las vicisitudes históricas y a la deplorable situación mental en la que se encuentran, las hacen tal vez efímeras, destinadas a una próxima desaparición ante las crecientes exigencias de la civilización y la concurrencia material intensiva de las corrientes migratorias que comienzan a invadir nuestra tierra. (Cunha, 2003, p. 17, énfasis propio)

Además de su racismo epistémico, los extrañamientos de Euclides y de los demás intelectuales identificados como blancos en esa época pueden ser leídos en la clave de lo que Grada Kilomba (2019) llamó de "otredad", es decir, "desemejanza", en reciente estudio en el campo de la psicoanálisis. El sujeto negro y, en este caso, aquí aplico al mestizo sertanejo, por ser fruto de la mesti- 
zación genética entre el indio, el negro africano y el "blanco" portugués (ancestralmente mestizo), no sólo es el "otro/otra" diferente y medido por el "yo" del blanco, sino la "encarnación de aspectos represivos del 'yo' del sujeto blanco". Más allá del concepto usual de alteridad, por tanto, se marca la "representación mental de lo que el sujeto blanco no quiere parecer" (Kilomba, 2019 , p. 38). Se observa que, aunque muchos referentes fueron del nordeste, esto no garantizó una ruptura con ese pensamiento racialista por simple afinidad al territorio.

Desde el siglo XIX hasta principios del siglo XX, las antiguas Facultades de Medicina de Bahía y de Río de Janeiro fueron las mayores productoras de tesis médicas que contenían factores psicológicos (Maio, 1995). En la primera estudió Nina Rodrigues, que logró elevar la medicina legal a la condición de especialidad científica inspirando, en la década de 1930, la fundación de la institución con su nombre por los colegas Afrânio Peixoto y Arthur Ramos. Su racismo epistémico se internacionalizó a través de artículos científicos desde 1897, basados en el darwinismo social y la eugenesia. Se extendió por las ciencias sociales, las investigaciones etnográficas de la cultura afrobrasileña y los análisis del pensamiento social, abordando raza, degeneración, atavismo, locura y nación en la República Vieja para definición de la identidad brasileña. Su caso es particular también porque describió cultos, prácticas y entidades sagradas de africanos y descendientes, imprimiendo valor etnográfico interesado en el fenómeno del mesianismo, además de pionero en cuanto a religiosidad de "negros y pardos", o sea, de los mestizos (Dalgalarrondo, 2007; Maio, 1995; Oda, 2001).

Al estudiar las causas y condiciones degenerativas de la población de la pequeña localidad de Serrinha -sertão de Bahia-, por ejemplo, escribió ser el "tipo medio general de la provincia o del estado" bahiano, completando con la capacidad social a través del examen de capacidad biológica escalada en la historia clínica. Bajo nuestra mirada del siglo XXI, más allá de los estudios biológicos de entonces, es impactante por la normalización lingüística reforzada por una teoría y juicio morales, como por ejemplo "pueblo semi-bárbaro", "contaminado por la tara hereditaria", "padre degenerado", "fuertemente histérico", "verdaderas monstruosidades" o "dado a luz un monstruo" (Rodrigues, 2008).

Al mismo tiempo que los constructos en torno a la degeneración y la eugenesia fueron asimilados por los psiquiatras planteando una amenaza social a la prosperidad, se presentaba también la solución con apoyo de los nacionalistas en un intento de escapar del pesimismo racial. Hay que recordar que el concepto de degeneración, introducido por el francés Benedict Morel en 1857, indicaba componentes biológicos y genéticos para las enfermedades mentales asociados con otros factores de fuerte carácter moral, como religiosidad y alcoholismo - 
génesis hasta el momento buscadas por las neurociencias (Caponi, 2014)-. La colonialidad interna también se nota ahí por el racismo, el sexismo y el clasismo rizomáticos. Al proclamar que la "fusión casi inviable de civilizaciones muy desiguales" produjo el jagunço, no "cualquier mestizo brasileño", sino propiamente este "mestizo del sertão", relata que él:

Supo acomodar las cualidades viriles de sus ancestros salvajes, indios o negros, a las condiciones sociales de la vida libre y a la civilización rudimentaria de los centros que habita. Muy diferente es el mestizo de la costa que el aguardiente, el ambiente de las ciudades, la lucha por la vida más intelectual que física, una civilización superior a las exigencias de su organización física y mental, debilitaron, abastardaron, acentuando la nota degenerativa que ya resulta del simple cruce de razas antropológicamente muy diferentes, y creando, en una regla general que conoce muchas excepciones, esos tipos inútiles y sin virilidad que van desde los degenerados inferiores, verdaderos productos patológicos, hasta esos talentos tan fáciles, superficiales y verborrágicos como abúlicos e improductivos, en los que los destellos de una inteligencia vivaz y de corto vuelo corren en paralelo con la falta de energía y hasta el perfecto equilibrio moral. (Rodrigues, 2008, p. 49, énfasis propio)

La concepción de enfermedad mental está directamente opuesta a la de salud mental y como producto de la dicotomía y fragmentación cartesianas, determinismos biológico y racial. Se destaca que el bandolerismo del sertão del nordeste (nombrado "cangaço" y sus "cangaceiros"), es otro buen caso que ejemplifica estas teorías. Conforme los prontuarios médicos, estaría lleno de "grandes flacos" y por lo tanto habría elevada propensión a la esquizofrenia, además de servir a los test en evidencia. Basándose en Cesare Lombroso, doctrinario del evolucionismo aplicado a los criminales, Rodrigues explicaba un "atavismo" físico y mental por "gérmenes de un pasado ancestral dormido en nuestra herencia", que sería comprobado con datos antropométricos, craneometría y frenología (Maio, 1995, p. 230).

Del prefacio de Arthur Ramos (2006, p. 18) de su obra As Raças Humanas e a Responsabilidade Penal no Brasil (1894), dedicada a los extranjeros, se destacan dos puntos: el primero es sobre la reorientación de sus estudios a las causas sociales y psicológicas que provocaban el "comportamiento asocial". En este sentido, atribuido al hecho de ser sorprendido por la ausencia de los signos de degeneración proferidos por la escuela italiana al examen antropométrico de la cabeza de Antonio Conselheiro (abordado adelante). Y el segundo, la consideración de esta obra como el "punto de partida indispensable para los estudios sobre la psicología social del negro brasileño" que, sin embargo, poco se menciona este marco. 
Es posible percibir el marcador de género en el "cangaço" sólo más recientemente, extrayéndose del trabajo de Adriana Negreiros (2018, p. 140) cómo las mujeres del bando fueron racializadas e hipersexualizadas por descripciones estéticas idealizadas, descritas con "hipergenitalismo" y objetificadas sexualmente. El psiquiatra Ranulfo Prata sería "definitivamente racista” y "poco empático con las mujeres", además de referirse a uno de los hombres como teniendo un "rostro bestial" y dotado de una "fealdad espantosa". Todavía era complaciente con los hombres, teniendo en cuenta que el caso de la violación colectiva de una anciana por parte de los bandoleros se trataría de "peraltes insignificantes" (p. 141). Estacio de Lima, otro psiquiatra, a pesar del desacuerdo en puntos de las teorías en boga, se extasiaba con las cabezas decapitadas por la policía para el análisis científico en la referida Escuela. Sobre la cabeza del bandolero "Volta Seca" demostró: "Busquemos, antes, el alma turbulenta del adolescente, o preadolescente, que las maldades del mestizo" (p. 140). Por el contrario, en actuación en el sureste, la inusual psiquiatra Nise da Silveira ya defendía la igualdad salarial entre géneros en 1927 y, en ese contexto, el mismo nordeste -concebido de manera totalitaria como retrasadoelegía Luíza Alzira Soriano como la primera alcaldesa de América Latina.

\section{Mesianismo, genocidio y psicopatología: los casos de Pedra Bonita y Canudos}

Hasta la década de 1960 muchos estudios se interesaron esencialmente por dos temas: a) el confrontamiento entre la psiquiatría y la religión; y b) la esclavitud relacionada con la psicopatía (Lima y Holanda, 2010). En el entendimiento de la ciencia, las culturas negras politeístas y animistas eran asociadas con la pobreza y el mestizaje, y también ubicadas en una etapa más "primitiva" de "desarrollo". Varios casos de análisis etnopsicológicos sobre el carácter místico-religioso asociado al fanatismo y a la "psicopatía" se identifican en el nordeste en ese momento histórico de convulsiones sociales, como los episodios de Pedra Bonita (1836-1838) - ciudad actual de São José do Belmonte- sertão de Pernambuco, seguido de Canudos (1896-1897) - antigua Monte Santo- sertão de Bahia. En el primero, decenas de personas murieron y, en el segundo, total destrucción y unos veinte mil muertos, además de los otros millares del ejército de Brasil, entendiéndose más claro por los análisis como un genocidio estatal.

En ambos casos, se asocia el fenómeno del mesianismo. Aunque comúnmente asociado al ámbito de la religiosidad, se añade una dimensión política, y puede ser entendido como la creencia fantástica en un mesías liberador que llegará o regresará reencarnado en figuras cristianas o mitos paganos, con el poder de revertir el orden social imperante donde prevalecen injusticias sociales, transformando las dolorosas condiciones de existencia. En los movimientos mesiáni- 
cos suele haber claramente un líder religioso o profano interesado en la justicia y la felicidad asociadas a aspectos sociales, políticos y religiosos. Existían en América del Norte, África, Europa y Brasil y, en el caso del nordeste, en general, con el tipo "rústico", reunió adeptos en condiciones modestas, familias, agricultores, labradores y vaqueros (Clemente, 2012, p. 86; Moura, 2021). Así, la influencia de la creencia ibérica en Don Sebastián, legendario rey portugués desaparecido en la batalla de Alcácer-Quibir/Marruecos (1578), originó el fenómeno llamado "sebastianismo" ( $\mathrm{y}$, en consecuencia, un "movimiento sebastiánico"), que se extendió por todo Brasil en el siglo XIX, como en estos casos. Una unión entre el fanatismo religioso, las ideas socialistas, los símbolos y la imaginación popular en el interior del nordeste, según Lúcia Gaspar (2006/2009).

En la obra de Euclides se observan los argumentos epistemológicos y términos utilizados por los científicos del área, especialmente por Nina Rodrigues. Entre los muchos estudios referentes a los hechos en Canudos, Fernández (2013, p. 197) en un análisis crítico, indica que Euclides en la sección "El hombre" presenta el argumento racista de la locura planteando a lo largo de la obra la necesidad de encauzar o disolver los "compatriotas retardatários". Así, el aislamiento del sertanejo, al margen de los procesos históricos y de las sociedades, impulsaría un condicionamiento de "psiques propensas a la sugestión y a la manipulación". En ese ambiente dado como anómalo, de un "cosmopolitismo escabroso" producido por la tradición colonial, el mesianismo fue atribuido al oriente y producto de otra de las "taras retardatarias" llevadas de Portugal a Brasil.

Éstos eran signos de la fatalidad de las leyes biológicas, como puntúa, y de una "mestización extremada", que significaba un "retroceso". Considerando que, para Euclides, en perspectiva temporal el tipo mestizo -que no le tocaba- tuvo una "breve existencia individual", "comprimiendo" en sí mismo "esfuerzos seculares" pero sin haber "luchado" y "constituciones mentales opuestas" y "diversas", resultaba casi siempre como un "desequilibrado". Atestiguando su determinismo racial: "todo hombre es ante todo una integración de esfuerzos de la raza a la que pertenece y su cerebro una herencia". Por lo que cuestionó: "¿cómo entender la normalidad del tipo antropológico que aparece, de repente, agrupando tendencias tan opuestas?". Con tono fatalista, el "sertanejo del norte" (referiente a esta región) era "innegablemente el tipo de una subcategoría étnica ya constituida” (Cunha, 2003, p. 110-112).

João Santos, el líder de Pedra Bonita, había proclamado el descubrimiento de un espacio sagrado con riquezas celestiales, llamando la atención el hecho de que los seguidores también serían blanqueados. Aunque ya se sabe del uso de 
un tipo de droga natural compartida en esta ocasión, me parece interesante destacar cuáles son los contenidos de los delirios o alucinaciones que aparecían. Como fenómenos pasibles de interpretación, a la luz de la historicidad y el contexto material/subjetivo de la gente, se indica la idealización de ascensión social bajo colonialidades intersubjetivas porque raza/color de la piel siempre ha sido un elemento de clasificación y de distinción social en el sur global colonizado. Al famoso líder de la comunidad de Canudos, Antonio Conselheiro, a la luz de la psiquiatría le fue atribuido el diagnóstico de "delirio crónico de Magnan" (Dalgalarrondo, 2007) y, si bien podría ser portador de un transtorno psicótico, es interesante notar que:

No sería un loco cualquiera, sino un profeta loco, mestizo, como las masas que le seguían. El mestizaje aportaría la predisposición a la enajenación mental, la sugestionabilidad y el desequilibrio mental necesarios para el contagio vesicular y daría (casi literalmente) el color brasileño a los fenómenos que los estudiosos de la psicología de las multitudes habían descrito en Europa. (Oda, 2008, p. 143, énfasis propio)

Las epidemias de locura, por su propio nombre, tendrían un carácter transmisible, pero durante su "exposición" una buena parte sería "refractaria al mal" (Rodrigues, 2006, pp. 98-99). Bajo el formato de "locura violenta de las multitudes", resalto algunos puntos a partir de la concepción del autor sobre los dos casos: en cuanto a los "sujetos", la mayoría tendría una predisposición inherente; en cuanto a las "causas", más allá de una predisposición hereditaria como se le atribuyó en los estudios internacionales inicialmente con "límites primitivamente fijados"- incluiría agotamiento orgánico, miseria, intoxicaciones, vicios debilitantes, excesos varios; y, en cuanto al "contenido delirante", asumirían características generales a diferencia de los sistematizados con temas específicos de persecución y grandeza. Sobre Pedra Bonita, que calificó de "horrible hecatombe", todavía con menos registros y trabajos en la literatura médico-psicológica que Canudos, refiere:

Estamos en presencia de un caso en el que son tan manifiestos y evidentes los caracteres de la epidemia vesánica de fondo religioso, que sólo una completa ignorancia de la psicología mórbida puede justificar el rigor penal con el que fueron abatidos algunos de los supervivientes, y la violencia revelada por quienes se ocuparon de los procedimientos para estigmatizar la supuesta perversidad de estos fanáticos. Aquí, más que en cualquier otra circunstancia, el desarrollo de la desviación morbosa de esta población puede atribuirse rigurosamente a la exaltación del misticismo de una reunión de mestizos psicológicamente más equilibrados, mediante la evocación violenta de los sentimientos y creencias atávicas de las razas inferiores de las que habían surgido. (Rodrigues, 2006, p. 94, énfasis propio) 
$Y$-tras trazar la evidente asociación entre degeneración, enfermedad mental, mestizaje, religiosidad y, por tanto, la inferioridad territorial- recomienda en la nota a pie de página consultar una "monografía" que mejor describe el episodio de "locura epidémica" ${ }^{3}$. Débora Cavalcantes de Moura Clemente (2012), en su tesis que abordó el evento en el campo de la Literatura, se acercó a la obra, consultó archivos regionales y el nacional en Rio de Janeiro, cuando era la sede del Imperio. Concluyó que las representaciones, apropiaciones y prácticas sociales relacionadas fueron guiadas por marcadores como raza y clase, reforzadas por los arquetipos naturalistas y lenguaje científico de finales del siglo XIX y un encuadramiento psiquiátrico con respecto a los "sebastianistas" (pueblo). Pero, en el caso de los "potentados" (representantes de la Iglesia Católica, hacendados y militares), se validaron sus discursos con arquetipos románticos, notando el cientificismo europeo desde la creación literaria misma: imaginación, observación y comprobación.

También emblemático en el territorio, el caso relativo al sacerdote "Padre Cícero" en el interior de Ceará, elevado a "santo popular" por "curas y milagros", fue analizado como un "fenómeno colectivo con contornos delirantes". Recién a fines de la década de 1960 las "epidemias de enfermedad mental" encuentran comprensión de los "fenómenos culturales", iniciándose la "despatologización” del mesianismo en la psiquiatría brasileña (Dalgalarrondo, 2007). Ser sugestionable y desequilibrado bajo los parámetros diagnósticos facilitaría epidemias de locura colectiva sumados a elementos de religiosidad y violencia, pertinente la conciliación relacionada con otras disciplinas no exclusivamente mentales. Por cierto, los estudios analizados no discuten otros hechos como la lucha de clases, el "aquilombamento" (protección en grupos de resistencia llamados "quilombos" contra la esclavitud), o los porqués sobre la defensa de la monarquía. La narrativa médica añadió fuerza a los discursos que justificaban genocidios de mestizos con modos tradicionales y comunitarios, centrados en la tierra y la religión. Refiere Moura (2021, párr. 4):

Al reconocer a D. Sebastián como único monarca legítimo y como enviado divino, los seguidores de la Piedra del Reino [nombre actual del lugar de la Pedra Bonita, donde hay una gran manifestación cultural que rescata esta historia, observación mía] se convirtieron en una amenaza para la élite local, el Estado y la Iglesia, que extinguieron violentamente el movimiento. Para justificarse, las clases dominantes presentaron a los sebastianistas como "fanáticos, es decir, insumisos religiosos extremos y agresivos".

\footnotetext{
${ }^{3}$ La obra de Antônio Ático de Sousa Leite: “'Fanatismo Religioso. Memória sobre o reino encantado na comarca de Vila Bela, con un juicio crítico del Consejero Tristão de Alencar Araripe', $2^{\mathrm{a}}$ ed. por Solidônio Ático Leite, juez de fuera, 1898 (A. R.).” (Rodrigues, 2006, p. 94).
} 
Mientras la institucionalización de la sociología se dio en el país en los lejanos años 30 , con giros y contribuciones sobre el tema de la raza y la cultura, aún es necesario introducir y estimular este debate en las disciplinas de la salud. Notemos, también, que, en la actualidad, con las convulsiones sociales, creciente influencia de las iglesias neopentecostales, políticas neoliberales y conservadoras en América Latina, se retoma el uso vulgar de "mesianismo", por lo que es interesante fundamentar los discursos a tenor de nuestras colonialidades.

\section{CONCLUSIÓN}

La afirmación y defensa de sociedades democráticas y emancipadas prescinde de estrategias discursivas para enfrentar problemas estructurales histórica y geográficamente construidos, como es el caso del racismo. Para ello, se utilizó la genealogía como herramienta en este trabajo y epistemologías críticas, observando que el caso del sertão del nordeste es ejemplar del racismo epistémico: es un territorio subalternizado desde el punto de vista geográfico y también racial, y cuando fue concebido en obras literarias y científicas de gran impacto y alcance hasta la actualidad. Bajo estos aspectos, difería del ideal de civilización y, así, constituía un obstáculo en el contexto político a finales del siglo XIX y principios del XX, cuando las racionalidades se centraron en la construcción de una nueva identidad del país en línea con los parámetros eurocéntricos de modernidad.

Por lo tanto, se convirtió en el objeto privilegiado de teorías racialistas y de los instrumentos en boga asimilados por la psiquiatría, como se demostró, destacando los papeles de Nina Rodrigues y Euclides da Cunha, entre otros personajes de la constitución del campo psicológico, especialmente relacionados con la región. Se observaron colonialidades epistémicas con efectos perdurables del colonialismo, ilustradas sobre elementos característicos y acontecimientos en el territorio, como el mesianismo y el "cangaço", con enfoque en la salud mental.

Notablemente, dada la sub-utilización y escasez de producciones académicas en ese ámbito, se recomienda intensificar los estudios históricos e interdisciplinarios que integren obras y documentos con fines discursivos, dentro de los desafíos de la agenda actual sobre los temas de raza y racismo. Se espera contribuir a la visibilidad del territorio en el debate de la Reforma Psiquiátrica brasileña. Además, se llama la atención al término ampliado "epistémico" en relación al tipo de racismo en cuestión, pues contempla lo científico y el epistemicidio respecto a otras sociedades. Finalmente, caben tensiones continuas para miradas que promuevan la descolonización epistémica y produzcan revisiones de las disciplinas y campos de prácticas locales y globales. 


\section{REFERENCIAS}

Akotirene, Carla (2019). Interseccionalidade. Coleção Feminismos Plurais. Polén.

Albuquerque Júnior, Durval Muniz de (2009). A invenção do Nordeste e outras artes (4. ed.) Massangana Cortez.

Albuquerque Júnior, Durval Muniz de (2019). O Rapto do Sertão: a captura do conceito de sertão pelo discurso regionalista nordestino. Revista Observatório Itaú Cultural, 1(25), 21-35. https://issuu.com/itaucultural/docs/obs25_issuu_1_/2?ff

Almeida, Silvio (2019). Racismo Estrutural. Polén.

Bolívar, Francisco J. Flórez (n.d.). Representaciones del caribe colombiano en el marco de los debates sobre la degeneración de las razas: geografía, raza y nación a comienzos del siglo XX. https://core.ac.uk/download/pdf/11860957.pdf

Carta-Aberta contra o Racismo Sistêmico na Psiquiatria (2020, 1 julio). Mad in Brasil (Mad in Brasil, Trad.). https: //madinbrasil.org/2020/07/carta-aberta-contra-oracismo-sistemico-na-psiquiatria/

Caponi, Sandra (2014). Loucos e degenerados: uma genealogia da psiquiatria ampliada. Fiocruz.

Cesarino, Leticia (2017). Colonialidade Interna, Cultura e Mestiçagem: repensando o conceito de colonialismo interno na antropologia contemporânea. Rev ILHA, 19(2), 73-105. https: / / doi.org/10.5007/2175-8034.2017v19n2p73

Clemente, Débora Cavalcantes de Moura (2012). Representações da história da Pedra do Reino no romance O Reino Encantado (1878), de Araripe Jr. Tese de Doutorado inédita, Universidade Federal da Paraíba.

Cunha, Euclides da (2003). Os Sertões. Martin Claret.

Dalgalarrondo, Paulo (2007). Estudos sobre religião e saúde mental realizados no Brasil: histórico e perspectivas atuais. Rev Psiquiatria Clínica, 34(Sup. 1), 25-33. https: / /doi.org/10.1590/S0101-60832007000700005

Fanon, Frantz (2008). Pele negra, máscaras brancas. EDUFBA

Fanon, Frantz (2020). Alienação e liberdade: escritos psiquiátricos. Ubu Editora.

Fernández, Juan Manuel (2013). Os Sertões: un retrato de la locura colectiva. Literatura: Teoria, História, Crítica, 15(2), 181-210. http://www.scielo.org.co/pdf/lthc/v15n2/v15n2a09.pdf

Foucault, Michel (2012). Nietzsche, a genealogia e a história, parte II. En Roberto Machado (Org.), Microfísica do poder. Graal.

Freyre, Gilberto (1933/2006). Casa Grande \& senzala: Formação da família brasileira sob o regime da economia patriarcal (51 ed.). Global.

Gaspar, Lúcia (2006/2009). Sebastianismo no Nordeste brasileiro. Pesquisa Escolar Online. Fundação Joaquim Nabuco.

http: / / basilio.fundaj.gov.br/pesquisaescolar/index.php?option=com_content\&view= article\&id $=419 \&$ Itemid $=1$

Grosfoguel, Ramón (2011). La descolonización del conocimiento: diálogo crítico entre la visión descolonial de Frantz Fanon y la sociología descolonial de Boaventura de Sousa Santos. CIDOB. 
Grosfoguel, Ramón (2016). A estrutura do conhecimento racismo/sexismo epistêmico e os quatro genocídios/epistemicídios nas universidades ocidentalizadas ao do longo século XV. Revista Sociedade e Estado, 31(1), 25-49. https://doi.org/10.1590/S010269922016000100003

Hall, Stuart (2003). Da Diáspora: identidade e mediações culturais. Ed. UFMG.

Instituto Brasileiro de Geografia e Estatística - IBGE (2019). Pesquisa Nacional por Amostra de Domicílios Contínua (PNAD). https://biblioteca.ibge.gov.br/visualizacao/livros/liv101673_informativo.pdf

Íñiguez-Rueda, Lupicinio (2010). O conhecimento como forma de resistência: uma conversa com Lupicínio Íñiguez-Rueda. Interface - Comunicação, Saúde, Educação, 14(34), 693-703. https://dx.doi.org/10.1590/S1414-32832010000300018

Kilomba, Grada (2019). Memórias da Plantação: episódios de racismo cotidiano. Cobogó Editora.

Lima, Nísia Trindade (2009). Uma brasiliana médica: o Brasil Central na expedição científica de Arthur Neiva e Belisário Penna e na viagem ao Tocantins de Júlio Paternostro. História, Ciências, Saúde - Manguinhos, 16(supl. 1), 229-248. https://doi.org/10.1590/S0104-59702009000500011

Lima, Andrea de Alvarenga \& Holanda, Adriano Furtado (2010). História da psiquiatria no Brasil: uma revisão da producãa historiográfica (2004-2009). Revista Estudos e Pesquisas em Psicologia, 10(2), 572-595. https://www.epublicacoes.uerj.br/index.php/revispsi/article/view/8983

Maio, Marcos Chor (1995). Brazilian Physician Nina Rodrigues: Analysis of a Scientic Career. Cad. Saúde Públ., 11(2), 226-237. https://doi.org/10.1590/S0102311X1995000200006

Maldonado-Torres, Nelson (2009). A topologia do ser e a geopolítica do conhecimento. Modernidade, Império e Colonialidade. In Boaventura de Sousa Santos \& Maria Paula Meneses (Orgs.), Epistemologias do Sul (pp. 337-381). Editora Almeidina.

Marques, Tiago Pires (2018). Illness and the Politics of Social Suffering: Towards a Critical Research Agenda in Health and Science Studies. Revista Crítica de Ciencias Sociais, (spe 2018), 141-164. https://doi.org/10.4000/rccs.7763

Medeiros, Tácito (1999). Psiquiatria e Nordeste: um olhar sobre a história. Rev. Bras. Psiquiatr. 21(3), 177. https://doi.org/10.1590/S1516-44461999000300010

Melo, Walter (2004). Ulysses Pernambucano: o enamorado da liberdade. Clio-Psyché Programa de Estudos e Pesquisas em História da Psicologia. Mnemosine, 1(0),185192. https: //www.epublicacoes.uerj.br/index.php/mnemosine/article/viewFile/41354/pdf_23

Moura, Valdir José Nogueira de (2021, 18 de enero). A Colorida festa da Pedra do Reino de São José do Belmonte. Tok de História.

https://tokdehistoria.com.br/2021/01/18/a-colorida-festa-da-pedra-do-reino-desao-jose-de-belmonte/

Negreiros, Adriana (2018). Maria Bonita: sexo, violenncia e mulheres no cangaço (1. ed.). Objetiva. 
Oda, Ana Maria Galdini Raimundo (2001). A teoria da degenerescência na fundação da psiquiatria brasileira: contraposição entre Raimundo Nina Rodrigues e Juliano Moreira. Psychiatry on line Brasil, 6(12). http://www.polbr.med.br/ano01/wal1201.php

Oda, Ana Maria Galdini Raimundo (2008). Nina Rodrigues e a loucura epidêmica de Canudos. Rev. Latinoam. Psicopat. Fund. 3(2), 139-144. https://www.scielo.br/pdf/rlpf/v3n2/1415-4714-rlpf-3-2-0139.pdf

Oliveira, Luciano Amaral (2011). "Mate um nordestino afogado" - Análise crítica de um artigo da Revista Época. Linguagem em (Dis)curso, 11(2), 361-376. https://www.scielo.br/pdf/ld/v11n2/08.pdf

Pinto, Clara Lúcia Martins de Souza (2017). Memória e subalternidade: representações do Nordeste em Patativa do Assaré. Dissertação de Mestrado inédita, Universidade Federal do Rio de Janeiro.

Polon, Luana (2019, octubre). Região Nordeste do Brasil. Estudo prático. https: //www.estudopratico.com.br/regiao-nordeste-do-brasil/

Prado Filho, Kleber (2017). A genealogia como método histórico de análise de práticas e relações de poder. Revista de Ciências HUMANAS, 51(2), 311-327. https://doi.org/10.5007/2178-4582.2017v51n2p311

Quijano, Aníbal (2005). Colonialidade do poder, eurocentrismo e América Latina. En Edgardo Lander (Org.), A colonialidade do saber: eurocentrismo e ciencias sociais. Perspectivas latino-americanas. (pp.107-130). CLACSO.

Quijano, Aníbal (2009). Colonialidade do Poder e Classificação Social. In Boaventura de Sousa Santos, \& Maria Paula Meneses (Orgs.), Epistemologias do Sul (pp. 73-117). Ed. Almeidina.

Ramos, Artur (2006). Prefácio. In Raimundo Nina Rodrigues (Org.), As coletividades anormais (pp. 10-10). Senado Federal, Conselho Editorial.

Rodrigues, Raimundo Nina (2006). As coletividades anormais. Senado Federal, Consetho Editorial.

Rodrigues, Nina (2008). Mestiçagem, degenerescência e crime. Hist. cienc. saudeManguinhos, 15(4), 1151-1182. https://www.scielo.br/pdf/hcsm/v15n4/14.pdf

Santos, Alessandro de Oliveira dos; Schucman, Lia Vainer \& Martins, Hildeberto Vieira (2012). Breve histórico do pensamento psicológico brasileiro sobre relações étnicoraciais. Psicologia: Ciência e Profissão, 32(spe), 166175. https://doi.org/10.1590/S1414-98932012000500012

Santos, Boaventura de Sousa (1995). Pela Mão de Alice (4. ed). Cortez Editora.

Santos, Boaventura de Sousa (2003). Entre Próspero e Caliban: colonialismo, póscolonialismo e inter-identidade. Novos Estudos CEBRAP, 66, 23-52. https: / /estudogeral.uc.pt/bitstream/10316/81691/1/Entre\%20Prospero\%20e\%20Calib an_colonialismo\%2C\%20pos-colonialismo\%20e\%20inter-identidade.pdf

Santos, Boaventura de Sousa (2020). Boaventura de Sousa Santos: na oficina do sociólogo artesão. Aulas magistrais 2011 - 2016. Edições Almeidina.

Santos, Ricardo Augusto dos (n. d). Oliveira Vianna, Eugenia e o Campo Intelectual da Primeira República. https://www.historia.uff.br/estadoepoder/6snepc/Mesas/mesa5-Ricardo.pdf 
Seixas, André Augusto Anderson; Mota, André, \& Zilbreman, Monica L. (2009). A origem da Liga Brasileira de Higiene Mental e seu contexto histórico. Revista de Psiquiatria do Rio Grande do Sul, 31(1), 82 https://doi.org/10.1590/S010181082009000100015

Wegner, Robert (2017). Dois geneticistas e a miscigenação: Octavio Domingues e Salvador de Toledo Piza no movimento eugenista brasileiro (1929-1933). Varia Historia, 33(61), 79-107. https://doi.org/10.1590/0104-87752017000100005

\section{EMILENE ANDRADA DONATO}

Profesora de la "Universidade Estadual de Ciências da Saúde de Alagoas" y Psicóloga, Brasil. Desarrolla estudios en los campos de evaluación de políticas públicas de salud y socio-histórico sobre salud mental. Experiencia en servicios y gestiones académica, técnica y de proyectos de educación y de salud. Estudiante del programa de doctorado "Persona y Sociedad en el Mundo Contemporáneo", de la Universitat Autònoma de Barcelona (España) y doctoranda visitante en el "Centro de Estudos Sociais" de la Universidade de Coimbra (Portugal).

edonatouncisal@gmail.com

https://orcid.org/0000-0001-5678-6426

\section{FORMATO DE CITACIÓN}

Donato, Emilene Andrada (2021). Racismo epistémico en el "sertão" del nordeste brasileño: un abordaje genealógico de la salud mental. Quaderns de Psicologia, 23(3), e1800. https://doi.org/10.5565/rev/qpsicologia.1800

\section{HISTORIA EDITORIAL}

Recibido: 20-02-2021

$1^{\text {a }}$ revisión: $12-04-2021$

Aceptado: 29-04-2021

Publicado: 25-01-2022 